Ann. Abeille, 1967, 10 (2), 83-95.

\title{
INFLUENGE DE L'UTILISATION DU PIÈGE A POLLEN SUR LE RENDEMENT EN MIEL DES COLONIES D’ABEILLES
}

\author{
P. LAVIL: \\ Station expérimentale d'Apiculture, \\ Centre de Recherches agronomiques du Sud-Est, 84-Montfavet \\ Institut national de la Recherche agronomique
}

\section{SOMMAIRE}

La présente publication concerne la récolte du pollen au moyen des trappes placées sur les ruches. Elle complète un mémoire publié en I963 (LAVIE et FRESNAYE). L'auteur a étudié principalement l'influence de l'utilisation du piège à pollen en apiculture pratique sur le rendement en miel. Les expériences ont été réalisées dans le sud-est de la France au cours de trois années : 1962, 1964, I 66 . Une centaine de colonies d'abeilles ont servi à réaliser l'ensemble des essais. Les principaux résultats obtenus vont toujours dans le même sens, à savoir :

$I^{\circ}$ la pose des trappes à pollen sur les ruches pendant 40 jours diminue la récolte et le rendement en miel (24, I p. Ioo en moyenne).

$2^{\mathrm{O}}$ Le rendement total en miel est moins affecté par la pose des trappes que la récolte elle-même.

$3^{\circ}$ En année favorable le poids de pollen récolté compense largement la perte subie sur le rendement en miel. En mauvaise année le pollen est récolté en faible quantité et le rendement en miel est plus bas que celui des témoins.

$4^{\circ}$ La pose des trappes à pollen n'occasionne pas de régression très sensible des surfaces de couvain (4,4 p. roo par rapport aux témoins).

$5^{\circ}$ Si l'on pose des trappes à pollen de mars à octobre sur des ruches, la récolte de pollen compense largement l'absence presque totale de récolte de miel.

En conclusion quelques conseils sont donnés aux apiculteurs qui désirent orienter leur exploitation vers la production du pollen.

\section{INTRODUCTION}

Les apiculteurs s'intéressent dans un but lucratif à la récolte du pollen butiné par l'abeille depuis environ une quinzaine d'années. Dès le début les apiculteurs se sont demandés à juste titre si la pose des pièges à pollen ne causait pas de préjudice à la colonie d'abeilles. Des objections à l'utilisation des trappes furent présentées mais peu d'essais complets furent effectués. De nombreux observateurs ont constaté que les abeilles étaient plus ou moins gênées par la présence des trappes mais ils se sont principalement attachés à étudier l'influence de celles-ci sur le développenıent de la colonie et du couvain, sur la réclusion des mâles et des reines, sur l'évacuation des déchets et sur la difficulté pour l'abeille à traverser le dispositif. 
En ce qui concerne l'influence sur la récolte du miel les renseignements précis sont rares. Les modèles de pièges à pollen et de grilles sont nombreux et la manière de les employer varie également selon les utilisateurs.

Depuis la création de la Station expérimentale d'Apiculture nous nous sommes intéressés à la récolte du pollen par l'abeille sous divers aspects. Des études concernant l'optimum de récolte du pollen au moyen des trappes supérieures ont été réalisées depuis I960 et nos principaux résultats ont fait 1'objet d'un mémoire (LAVIE et FRESNAYE, I963). L'importance quantitative de la récolte du pollen dans le Sud-est n'est sans doute pas étrangère au développement de nos recherches dans ce sens. I a récolte moyenne par ruche est en effet beaucoup plus élevée que celles qui furent signalées par d'autres auteurs en France ou à l'étranger.

Chauvin, dès I955, préconise la tôle perforée à trous ronds pour le passage des butineuses dans le piège et un passage supplémentaire entièrement libre à l'arrière de la ruche pour la sortie des mâles et l'évacuation des déchets. Il mentionne d'autre part que, dans la ruche avec trappe, l'élevage du couvain se trouve réduit, principalement si 1'on emploie des grilles à haut rendement du type Böttcher (r94I). Le temps pendant lequel est posée la trappe sur la ruche est un facteur très important à considérer et il influe sur le comportement de la colonie d'abeilles bien que la pose d'une trappe en continu soit possible dans certaines conditions expérimentales bien définies sans nuire à son développement. Fn ce qui concerne la gêne causée aux abeilles par le dispositif, Todn et BIshop (I940) et HirschFel,der (I95I) pensent que les ruches ne doivent pas conserver les trappes en service d'un bout à l'autre de la saison. Chauvin et certains observateurs français ayant récolté du pollen en quatité appréciable sont d'un avis plus nuancé. Nous mêmes, au cours d'expériences à Montfavet, avons placé à plusieurs reprises des pièges à pollen (avec ouverture à l'arrière pour les mâles) pendant un an ou deux sans interruption, ou pendant une très longue période allant du début de mars à la fin octobre. Nous n'avons pas enregistré de mortalité dans les colonies, soumises cependant à des conditions extrêmes. Dans de nombreux cas, les ruches se sont développées normalement et ont parfois récolté un surplus de miel. Il nous semble que ces différences d'opinions s'expliquent facilement. Les colonies américaines sont souvent très développées et les miellées ont une importance et une continuité souvent inconnues en Europe. Il est donc probable qu'une trappe gêne beaucoup plus les colonies d'abeilles aux États-Unis qu'en France. Nous avons d'ailleurs contrôlé indirectement ce point de vue (LAVIE et FRESNAYE, I963) : les ruches anormalement développées sont gênées par les pièges : elles périclitent, leur couvain régresse et elles récoltent moins de pollen et de miel que les ruches normalement fortes. Quant aux réserves de HIRSCHFEL,DER, elles sont justifiées du fait de l'emploi de plaques perforées spéciales qui imposent des conditions de prélèvement trop sévères aux abeilles.

Louveaux dès I954 a montré que les récoltes de pollen pouvaient être abondantes, même en dehors de toute miellée et qu'il n'y a aucun rapport entre les deux activités de récolte à un moment donné. Mais si l'on considère une saison toute entière, il est intéressant de savoir si, dans une région donnée, le rendement en miel est affecté ou non par la pose des trappes au cours de l'année. Les essais divers concernant la récolte du pollen que nous avons conduits pendant de nombreuses années nous ont amené à étudier de plus près l'influence de l'utilisation du piège à pollen sur la récolte et le rendement en miel de la ruche. Cette étude a été suivie sur trois années diffé- 
rentes : r962, r964 et I966. Nous avons essayé de répondre en partie à la question suivante : la récolte du pollen est-elle rentable compte tenu notamment de la baisse de rendement en miel qui peut en résulter?

\section{MATÉRIEL E'T MÉTHODES}

Les expériences ci-dessous décrites font suite à nos essais sur la récolte du pollen au moyen de la trappe supérieure (LAVIE et FrESNAYE I963); le matériel et les méthodes employés furent les mêmes. A part quelques particularités il est donc inutile d'y revenir ici en détail.

Rappelons cependant les points importants concernant la récolte du pollen. Le piège comporte une tôle perforée ayant trois rangées de trous de $0,5 \mathrm{~cm}$ de diamètre pour le passage des abeilles. La ruche Dadant Blatt possède une sortie arrière rendue libre quelques jours après la pose des trappes à pollen. Celles-ci sont nettoyées régulièrement à chaque visite pendant leur utilisation. Le pollen est récolté tous les 5 jours, ruche par ruche, pesé et séché à l'étuve pendant 24 heures. Toutes ces dispositions ont été adoptées à la suite des résultats expérimentaux précédents. L'emplacement expérimental dans le Gard est toujours le même, sauf en I 966 où il se trouvait à $\mathrm{I}, 500 \mathrm{~km}$ environ de l'ancien rucher, devenu inaccessible. 92 colonies furent utilisées au cours des essais : 32 en 1962,30 en I964 et 30 en I 966 suivant le calendrier du tableau I. Les ruches furent d'abord installées dans les garrigues à Cistes au moment de la pose des trappes et le pollen fut récolté pendant 40 jours environ sur la moitié des colonies, les autres servant de témoins. A cette époque les ruches étaient situées dans une zone offrant peu de cultures mellifères. En i 966 , par exemple, l'augmentation moyenne du poids des ruches témoins entre le $21 / 4$ et le $7 / 6$ a été de $5,200 \mathrm{~kg}$ ce qui est faible pour cette période. Par contre, l'emplacement est hautement pollinifère : les Cistes sont très abondants et l'abeille se polarise préférentiellement sur Cistus albidus dont le pollen représente $60 \mathrm{p}$. $100 \mathrm{du}$ total recueilli dans les pièges. Précisons que la récolte de pollen de Cistes fut faible en 1964 à cause des gelées ayant détruit ces plantes au cours de l'hiver 1962-1963. C'est d'ailleurs pour cette raison que les essais n'ont pas été poursuivis en 1963 ; leur reprise en 1964 correspond à un départ de végétation de nouvelles plantes. Il est donc normal que la récolte moyenne par ruche soit la moitié de celle que nous avions l'habitude d'obtenir les années précédentes dans le même biotope. Etant donné les résultats de I964, nous avons supprimé les essais en 1965 , la reprise de la végétation étant encore peu satisfaisante. En 1966, la floraison et la récolte du pollen étaient redevenues normales, les Cistes étant à nouveau bien développés.

Chaque année, après cette première partie de l'expérience la trentaine de ruches était transportée en altitude $(900 \mathrm{~m})$ dans le Vaucluse sur des cultures de lavandes et de lavandins afin d'y récolter du miel. Nous avons complété la notion de récolte de miel employée généralement par de nombreux auteurs par celle de rendement en miel qui est à notre sens plus précise. L'évaluation de la récolte de miel est en effet insuffisante comme nous le verrons plus loin à la lecture des tableaux de résultats. Le rendement moyen (en $\mathrm{kg}$ ) d'une ruche (R) est obtenu au moyen de la formule suivante :

$$
\mathrm{R}=\mathrm{MH}+\mathrm{MC}+\left(\mathrm{Pr}-\mathrm{P}_{2}\right)-\mathrm{N}
$$

MH étant le poids du miel extrait de la (des) hausse (s),

MC le poids du miel extrait des cadres de corps de ruche (s'il y a lieu)

PI le poids de la ruche à l'hivernage précédent l'expérience,

$\mathrm{P}_{2}$ le poids de la ruche à l'hivernage suivant l'expérience,

$\mathrm{N} \quad$ le poids du sucre ou du miel donné en nourrissement (s'il y a lieu).

Des pesées hebdomadaires pendant toute l'expérience ont permis de recueillir des renseignements complémentaires. La surface du couvain de chaque ruche a été évaluée en points, avant et après la pose des trappes, d'après la méthode que nous employons habituellement (FRESNAYE I962). Chaque point correspond à $40 \mathrm{~cm}^{2}$ de couvain.

Parmi les facteurs météorologiques c'est le vent violent (mistral), qui influe le plus directement sur les récoltes de pollen. Nous avions déjà remarqué en 1960 et I96r cette action directe du vent dans nos essais. Le tableau 2 résume sommairement quelques facteurs météorologiques des trois années d'essais et ceux de l'année ig6r. Si le vent a une action le jour même où il souffle, il n'influence pas la récolte moyenne de pollen pendant toute la période des essais. Le vent s'est manifesté normalement au cours des différents essais notamment au cours des années 1962 et 1966. D'ailleurs le mistral souffle assez violemment chaque année à cette époque dans le sud-est. Le vent peut également être un facteur limitant pour le butinage lors de la miellée des lavandes et des lavandins. Le tableau 2 nous indique encore que les périodes très pluvieuses ou très sèches pendant les essais n'influent guère sur la récolte du pollen : par exemple i961 et 1966. La durée des périodes de beau temps ensoleillé n’a 


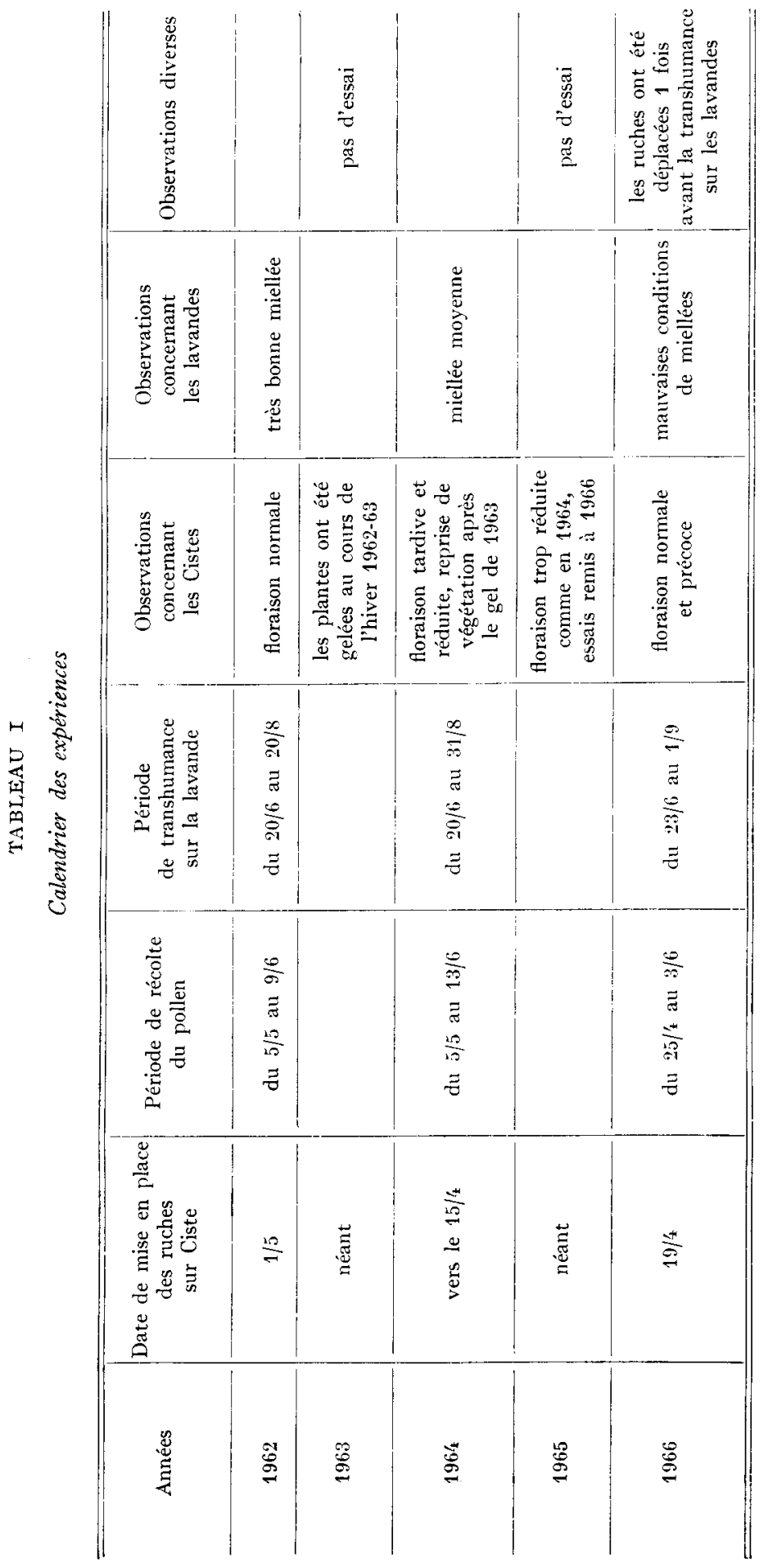




\section{TABLEAU 2}

Tableau résumé de quelques facteurs météorologiques pendant la pose des trappes à pollen et la floraison des cistes

\begin{tabular}{|c|c|c|c|c|c|c|c|}
\hline Année & $\mid \begin{array}{c}\text { Durée de } \\
\text { l'expérience } \\
\text { en jours }\end{array}$ & $\begin{array}{l}\text { Jours } \\
\text { de beau } \\
\text { temps }\end{array}$ & $\begin{array}{l}\text { Jours } \\
\text { de temps } \\
\text { nuageux } \\
\text { sans pluie }\end{array}$ & $\begin{array}{l}\text { Jours } \\
\text { de pluie }\end{array}$ & $\begin{array}{c}\text { Jours } \\
\text { de vent } \\
\text { "mistral" }\end{array}$ & $\begin{array}{c}\text { Pollen } \\
\text { récolté } \\
\text { par ruche } \\
\text { en } \mathrm{kg}\end{array}$ & Observations \\
\hline 1961 & 44 & 10 & 6 & 13 & $\begin{array}{c}15 \\
\text { (dont } 9 \\
\text { violents) }\end{array}$ & 5,430 & $\begin{array}{l}\text { Saison très ventée } \\
\text { et orageuse. }\end{array}$ \\
\hline 1962 & 40 & 14 & 6 & 7 & $\begin{array}{c}13 \\
\text { (dont } 4 \\
\text { violents) }\end{array}$ & 5,115 & $\begin{array}{l}\text { Saison ventée et } \\
\text { sèche. }\end{array}$ \\
\hline 1964 & 44 & 20 & 11 & 4 & $\begin{array}{c}9 \\
\text { (dont 5 } \\
\text { violents) }\end{array}$ & 2,698 & $\mid \begin{array}{l}\text { Tenir compte du } \\
\text { fait que les cistes } \\
\text { ont gelé en } 1963 \text {. } \\
\text { Saison peu ventée } \\
\text { mais sèche. }\end{array}$ \\
\hline 1966 & 45 & 24 & 6 & 3 & $\begin{array}{c}12 \\
\text { (dont } 4 \\
\text { violents) }\end{array}$ & 4,454 & $\begin{array}{l}\text { Saison ventée et } \\
\text { sèche. }\end{array}$ \\
\hline
\end{tabular}

également aucune influence sur la récolte moyenne de chaque ruche. Le facteur le plus important qui limite la récolte de pollen n'est donc pas un facteur météorologique agissant directement pendant la floraison ; c'est plutôt un facteur déterminant l'absence des fleurs ou l'épanouissement défectueux de celles-ci. Par exemple, les Cistes ayant été gelés auparavant, en I $^{6} 64$ la récolte de pollen fut faible et cela malgré 20 jours de beau temps, très peu de pluie diurne et seulement 5 jours de mistral violent. De toute manière chaque année les conditions sont identiques pour les ruches munies de trappes et pour les témoins et il n'est guère utile d'analyser les facteurs météorologiques plus à fond dans ce type d'expérience.

Précisons encore que toutes les ruches sont disposées au hasard dans le rucher afin d'éviter les phénomènes de dérive.

\section{RÉSULTATS}

Les résultats portent sur trois essais que nous analyserons successivement, mais pour la clarté du texte nous examinerons plus en détail l'année Ig66 seule. En effet, il n'existe pas de différence fondamentale entre les résultats obtenus au cours des trois saisons considérées. En I966 d'autre part les précisions sont plus nombreuses dans les relevés ce qui est dî au perfectionnement constant des protocoles d'expériences d'année en année. Enfin I966, de même que I962, offre une situation plus normale que I964 en ce qui concerne la floraison des Cistes et la récolte du pollen. L'année I 964 dont les chiffres vont dans le même sens, mais qui présente des anomalies, sera prise en considération avec prudence et seulement dans les résultats globaux.

Les tableaux 3,4 et 5 exposent les résultats détaillés obtenus au cours des trois années intéressées et le tableau 6 établit la comparaison entre les trois essais et les moyennes générales obtenues. Leur lecture dispense d'explications complémentaires 


\section{TABLEAU 3}

Résultats de l'année 1962

\begin{tabular}{|c|c|c|c|c|c|c|c|}
\hline & 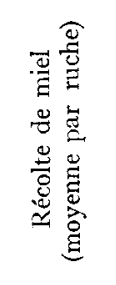 & 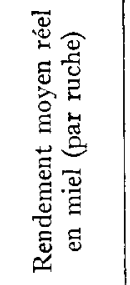 & 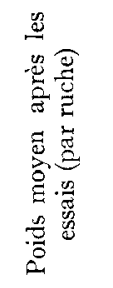 & 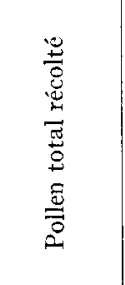 & 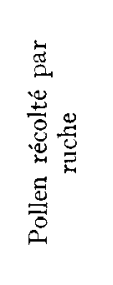 & 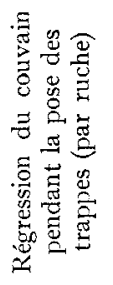 & 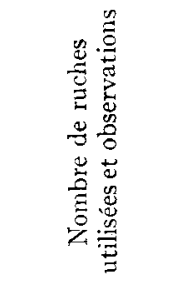 \\
\hline Ruches témoins $\ldots$ & 22,28 & 29,15 & 33,46 & 0 & 0 & 51 & $\begin{array}{l}16 \text { ruches dont } \\
2 \text { ruches } \\
\text { atteintes de } \\
\text { loque }\end{array}$ \\
\hline $\begin{array}{c}\text { Ruches avec trappes } \\
\text { à pollen } . . . . . .\end{array}$ & 16,78 & 25,41 & 33,75 & 81,845 & 5,115 & 67,6 & 16 ruches \\
\hline $\begin{array}{c}\text { Gain ou perte pour } \\
\text { les ruches avec } \\
\text { trappes par rap- } \\
\text { port au témoin. }\end{array}$ & $-5,500$ & $\begin{array}{c}-3,74 \\
-12,8 \%\end{array}$ & $+0,290$ & $+81,845$ & $+5, \mathbf{1 1 5}$ & $-16,6$ & \\
\hline
\end{tabular}

TABLEAU 4

Résultats de l'année 1964

\begin{tabular}{|c|c|c|c|c|c|c|c|}
\hline & 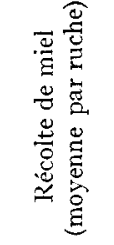 & 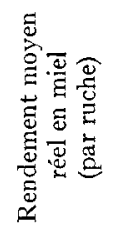 & 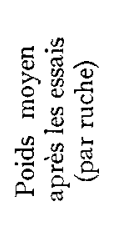 & 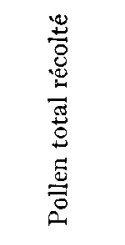 & 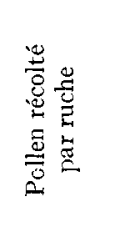 & 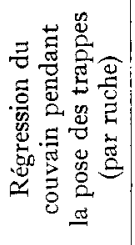 & $\begin{array}{l}\text { Nombre de } \\
\text { ruches utilisées } \\
\text { et observations }\end{array}$ \\
\hline Ruches Témoins .. & 19,128 & 23,798 & 39,464 & 0 & 0 & 59,4 & 1't ruches \\
\hline $\begin{array}{l}\text { Ruches avec trappes } \\
\text { à pollen } \ldots . . . .\end{array}$ & 12,850 & 19,750 & 39,821 & 40,470 & 2,698 & 67,7 & 15 ruches \\
\hline $\begin{array}{c}\text { Gain ou perte pour } \\
\text { les ruches avec } \\
\text { trappes par rap- } \\
\text { port au témoin }\end{array}$ & $-6,278$ & $\begin{array}{l}-4,048 \\
-17 \%\end{array}$ & $+0,357$ & $+40,470$ & $+2,698$ & $-8,3$ & $\begin{array}{c}1 \text { ruche } \\
\text { orpheline } \\
\text { (avec trappe) }\end{array}$ \\
\hline
\end{tabular}


TABLEAU 5

Résultats de l'année 1966

\begin{tabular}{|c|c|c|c|c|c|c|c|}
\hline & 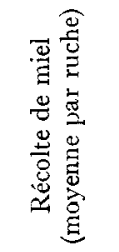 & 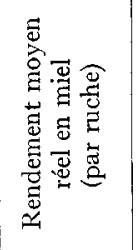 & 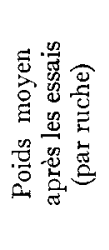 & 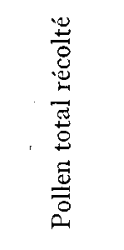 & 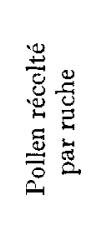 & 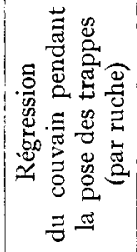 & $\begin{array}{l}\text { Nombre de } \\
\text { ruches utilisées } \\
\text { et observations }\end{array}$ \\
\hline Ruches témoins .. & 19,780 & 24,000 & 33,266 & 0 & 0 & 29,87 & 15 ruches \\
\hline $\begin{array}{c}\text { Ruches avec trappes } \\
\grave{a} \text { pollen } \ldots \ldots \ldots\end{array}$ & 9,233 & 13,800 & 28,000 & 81,823 & 4,454 & 35,07 & 15 ruches \\
\hline $\begin{array}{c}\text { Gain ou perte pour } \\
\text { les ruches avec } \\
\text { trappes par rap- } \\
\text { port au témoin. }\end{array}$ & $-10,457$ & $\begin{array}{l}-10,200 \\
-42,5 \%\end{array}$ & $-5,266$ & $+81,823$ & $+4,454$ & $-5,20$ & $\begin{array}{c}3 \text { ruches } \\
\text { avec un peu } \\
\text { de mycoses } \\
\text { (avec trappes) }\end{array}$ \\
\hline
\end{tabular}

TABIEAU 6

Comparaison des résultats des trois essais

\begin{tabular}{|c|c|c|c|c|}
\hline \multirow{2}{*}{ Moyenne par ruche } & \multicolumn{3}{|c|}{ Année } & \multirow{2}{*}{$\begin{array}{l}\text { Moyenne générale } \\
\text { des trois années }\end{array}$} \\
\hline & 1962 & $196^{\prime}$ & 1966 & \\
\hline $\begin{array}{l}\text { Rendement en pollen (en } \\
\mathrm{kg}) \ldots \ldots \ldots \ldots \ldots \ldots\end{array}$ & 5,115 & 2,698 & $5,45 x$ & 4,422 \\
\hline $\begin{array}{r}\text { Perte de récolte en miel } \\
(\text { en } \mathrm{kg}) \\
\ldots \ldots \ldots \ldots \ldots\end{array}$ & 5,500 & 6,278 & 10,547 & $7,4 \pm 1$ \\
\hline $\begin{array}{l}\text { Perte de rendement réel } \\
\text { en miel (en } \mathrm{kg}) \ldots \ldots \ldots\end{array}$ & $3, \pi / 4$ & 4,$0 ; 8$ & 10,200 & $\begin{array}{c}6,329 \\
\text { soit } 24,1 \%\end{array}$ \\
\hline $\begin{array}{c}\text { Perte de couvain (en } \\
\text { points) des ruches avec } \\
\text { trappes par rapport aux } \\
\text { témoins } \ldots \ldots \ldots \ldots \ldots\end{array}$ & 16,6 & 8,3 & 5,2 & 10,03 \\
\hline $\begin{array}{r}\text { Pertes diverses dans le } \\
\text { cheptel avec trappes }\end{array}$ & néant & $\begin{array}{l}1 \text { ruche } \\
\text { orpheline }\end{array}$ & $\begin{array}{l}3 \text { attaques } \\
\text { mycoses } \\
\text { très légères }\end{array}$ & $\begin{array}{l}1 \text { ruche perdue } \\
\text { sur } 46\end{array}$ \\
\hline $\begin{array}{l}\text { Pertes diverses chez les } \\
\text { témoins } \ldots \ldots \ldots \ldots \ldots \ldots\end{array}$ & $\begin{array}{l}2 \text { ruches } \\
\text { avec loque } \\
\text { américajne }\end{array}$ & néant & néant & $\begin{array}{l}2 \text { ruches loqueuses } \\
\text { sur } 45 \text {. }\end{array}$ \\
\hline
\end{tabular}


et l'on s'aperçoit très rapidement que le fait de placer des pièges à pollen sur les ruches gêne la récolte et fait baisser le rendement en miel au cours de la saison incriminée. On constate d'autre part qu'une grande différence existe entre la récolte et le rendement en miel et que la pose des trappes affecte plus la récolte du miel que le rendement final de la colonie d'abeilles. Par contre, il n'existe pas de relation entre le rendement en pollen et la baisse de rendement en miel; par exemple si l'on compare I962 et I964 on peut voir qu'une récolte inıportante de pollen n'entraîne pas obligatoirement une perte de rendement en miel accrue. La valeur mellifère de l'année peut modifier les résultats dans une certaine mesure : il semble que les récoltes de miel soient nivelées pour les deux lots de ruches lorsqu'il y a abondance de nectar tandis qu'en année peu mellifère les ruches témoins sont favorisées par rapport aux récolteuses de pollen. Par exemple en I 962 , année très mellifère, le rendement en miel diminue de i2,8 p. Ioo dans les ruches avec trappes tandis qu'en 1966, où la récolte est plus faible, ce chiffre atteint 42,5 p. IOO. Nous pouvons constater encore pour les ruches avec pièges qu'en mauvaise année mellifère la perte de récolte est voisine de la perte de rendement. L,e tableau 6 nous indique également qu'il n'y a pas de rapport direct entre la réduction des surfaces de couvain et la perte de rendement en miel. En effet, en I962, oì les ruches avec trappes ont perdu I2,8 p. Ioo de leur rendement en miel, elles ont perdu le maximum de points de couvain par rapport aux témoins (I6,6). En I966, avec une perte de rendement en miel de 42,5 p. Ioo, elles voient leur couvain régresser seulement de 5,2 points par rapport aux témoins.

On trouve en définitive que la perte de rendement en miel sur l'ensemble do trois années est de 24,1 p. 100 pour une récolte moyenne de 4,422 kg de pollen par ruche. Si l'on considère seulement les deux années normales, I962 et I966, (celles où un apiculteur aurait posé ses trappes) la perte de rendement en miel est de 27,65 p. 100 pour une récolte moyenne de $5,284 \mathrm{~kg}$ de pollen par ruche.

Nous avons remarqué encore que les ruches ayant servi à la récolte du pollen sont légèrement plus lourdes que les témoins au moment de l'hivernage suivant : $0,290 \mathrm{~kg}$ en I962 et $0,357 \mathrm{~kg}$ en I964. Cependant, en I966 ce sont les ruches témoins qui ont le mieux préparé l'hivernage puisque les ruches avec trappes accusent $5,266 \mathrm{~kg}$ de moins. Nous n'avons actuellement aucune explication à donner en ce qui concerne ces résultats diamétralement opposés.

A d'autres points de vue nous n'avons guère trouvé de différences entre témoins et colonies récolteuses de pollen. Certaines ruches ont changé leur reine, d'autres sont devenues orphelines et d'autres ont été atteintes de maladies du couvain sans qu'il soit possible d'incriminer les trappes. Toutefois, l'apparition de trois cas de mycoses a eu lieu dans des ruches munies de trappes.

Le tableau 7 indique l'évolution des surfaces de couvain dans les ruches. Les mesures effectuées avant la pose et après l'enlèvement des pièges permettent de connaître la régression ou l'augmentation des surfaces de couvain pendant les essais. A cette période de l'année et à cet emplacement les surfaces de couvain régressent de 23,5 p. 100 en moyenne qu'il s'agisse des témoins ou des autres ruches. Cette diminution de la surface de couvain n'est d'ailleurs pas la même tous les ans ; par exemple, en I966, elle a été plus faible (I6,9 p. I0o). On remarquera sur le tableau que les colonies qui possèdaient le plus de couvain avant les essais sont toujours celles où le couvain a regressé le plus, qu'il s'agisse des ruches témoins ou des autres. La comparaison entre la réduction de la surface de couvain chez les ruches témoins et celles qui ont eu des 
trappes à pollen est de loin la plus intéressante. Le couvain régresse toujours un peu moins dans les colonies témoins mais la différence est faible; 6,6 p. Ioo en Ig62, $3,8 \mathrm{p}$. Ioo en I964 et $2,6 \mathrm{p}$. Ioo en I966. Si l'on considère l'ensemble de l'expérience les ruches qui récoltent le pollen perdent en moyenne 4,4 p. 100 de couvain de plus que

TABLEAU 7

Évolutions des surfaces de convain

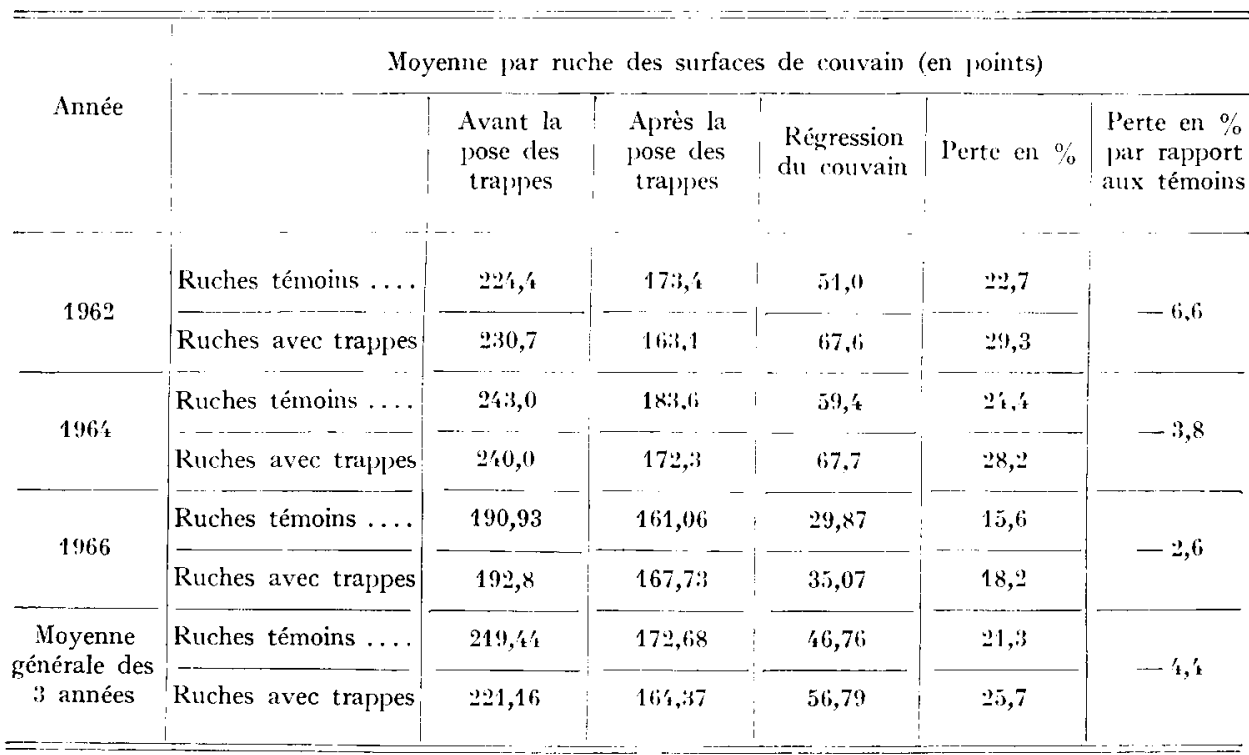

les témoins. Cependant, ce chiffre n'est pas suffisant pour expliquer la chute du rendement en miel qui est nettement plus élevée. Signalons encore que les groupes de colonies qui récoltent le plus de pollen ne sont pas obligatoirement ceux qui ont vu leur couvain diminuer le plus.

Si nous examinons les résultats de Ig66 de plus près, nous remarquons que les surfaces de couvain avant la pose des trappes sont inférieures à celles de r 962 et de Ig64 et que d'autre part la régression de la surface de ce couvain est plus faible au cours de l'essai. En I966, les ruches étaient moyennement fortes ce qui est plus favorable pour récolter le pollen que lorsque les ruches sont trop populeuses. I,es abeilles cette année là, se sont polarisées presque uniquement sur les Cistes et leur pollen a représenté au moment de la pleine floraison environ go p. Ioo du contenu des tiroirs dans la plupart des pièges. En général, les colonies qui récoltaient le plus de pollen étaient celles qui visitaient les Cistes. A l'inverse, cependant, une ruche a sans raison apparente recueilli très peu de pollen et jamais de pollen de Ciste. Le cas moyen est représenté par deux ruches dont la récolte est faible $(3,245 \mathrm{~kg}$ et $3,568 \mathrm{~kg})$ et dont le pourcentage de Ciste atteint seulement $60 \mathrm{p}$. Ioo. Comme chaque année la récolte du pollen a été irrégulière pendant l'essai et soumise aux facteurs météorologiques mais la moyenne sur l'ensemble de la saison est élevée. En effet trois colonies ont 
laissé dans les pièges plus de $200 \mathrm{~g}$ et dix colonies plus de $100 \mathrm{~g}$ de pollen comme moyenne journalière. La miellée par contre a été assez médiocre dans les emplacements successifs occupés par le rucher.

L'importance pondérale de la récolte du pollen par les abeilles dans le sud-est est encore confirmée par quelques expériences conduites à Montfavet en I 965 et en I 966 dans d'autres buts. Elles montrent d'ailleurs que la présence des cistes n'est pas indispensable pour amasser beaucoup de pollen car à Montfavet ces plantes sont très rares dans la zone de butinage du rucher utilisé. Au cours de detux saisons les colonies d'abeilles furent munies de trappes à pollen du début de mars à la fin d'octobre, c'est-à-dire pendant toute la période active des butineuses. En 1965, trois ruches ont récolté $10,934 \mathrm{~kg}$ de pollen en moyenne malgré le remérage de l'une d'elle en mai-juin et la présence d'un grand nombre d'autres colonies dans le rucher. Les abeilles n'ont pas été nourries et 1'une des ruches à récolté $7,6 \mathrm{~kg}$ de miel. En $\mathrm{r} 966$, seize ruches ont été traitées de la même manière et leur moyenne a atteint $8,761 \mathrm{~kg}$ de pollen par colonie. Nous devons tenir compte du fait que cette moyenne est abaissée par la présence de 8 colonies d'abeilles étrangères au sud-est et mal acclimatées. Si l'on choisit seulement les 8 colonies d'abeilles originaires de Montfavet, la récolte moyenne atteint $10,590 \mathrm{~kg}$ par ruche. Dans ce dernier lot, 4 ruches dépassent les I3 $\mathrm{kg}$ et l'une d'elle a récolté I6, I54 kg dans sa saison. Aucune de ces ruches n'a été nourrie, mais une seule a récolté du miel $(9 \mathrm{~kg})$. Il est donc possible dans certaines conditions de placer des pièges à pollen très longtemps sur les ruches sans les voir péricliter. Cependant le rucher placé à Montfavet est certainement favorisé pour ce type d'exploitation en raison de la présence de cultures et de prairies, irriguées pendant tout l'été. Des ruches sans trappes à pollen dans le même emplacement n'ont d'ailleurs pas récolté beaucoup de miel, mais les relevés précis à ce sujet nous manquent, les essais ayant été orientés dans d'autres directions.

\section{DISCUSSION DES RÉSULTATS}

Bien peu d'auteurs ont expérimenté dans le même sens que nous. Les travaux sont rares, souvent anciens et incomplets et les résultats sont divergents. Seul BARAC (I965) a étudié la question en détail.

Les Américains et les Allemands sont persuadés que la pose des trappes à pollen est défavorable et qu'elle entraine obligatoirement une baisse de rendement en miel importante. Mais ToDD et BISHOP (I940) ne précisent pas par des données chiffrées la gêne causée par les pièges à pollen. HirschFEI,DER (I95I) est déjà plus précis. Après avoir employé des grilles du type BötTchER, il pense qu'en compensation des pelotes laissées dans le piège, de nombreuses butineuses de nectar se consacrent à la récolte du pollen. Il en résulte une baisse de la récolte de miel d'environ $25^{\circ} \mathrm{g}$ par $\mathrm{kg}$ de pollen soustrait aux abeilles, mais cette récolte de miel n'est jamais annulée. Pour les trois auteurs précités les trappes à pollen ne doivent pas rester en place sur les ruches toutes la saison, sinon elles causent de graves dommages aux colonies. Il ne faut toutefois pas généraliser puisque nous-mêmes réalisons cette opération chaque année dans le sud-est de la France sans inconvénients sérieux pour les abeilles. 
L'opinion de Chauvin (I955) est différente. Pour cet auteur la trappe à pollen ne gêne nullement les abeilles dans des conditions correctes d'utilisation et de nombretux apiculteurs français sont de cet avis. Il est bon de préciser qu'il s'agit ici d'un piège confectionné grâce à une tôle perforée à trous ronds de $5 \mathrm{~mm}$ de diamètre.

RyBAKOV (I96I) affirme de son côté que la pose de pièges à pollen en Ukraine sur une dizaine de ruches augmente la récolte de miel. Il a trouvé que les colonies munies de ces dispositifs récoltaient en moyenne $40,9 \mathrm{~kg}$ de miel tandis que les colonies témoins en 'récoltaient seulement $35,5 \mathrm{~kg}$.

Très récemment BARAC (I965) a étudié les relations de cause à effet d'une manière plus détaillée sur une centaine de ruches au total pendant 3 saisons apicoles en Roumanie. Il en a déduit que le prélèvement du pollen au moyen de trappes n'a pas d'influence défavorable sur la production du miel. Voici le tableau qui résume les résultats obtenus :

\begin{tabular}{|c|c|c|c|c|}
\hline \multirow[b]{2}{*}{ Année } & \multicolumn{2}{|c|}{ Groupe témoin } & \multicolumn{2}{|c|}{ Groupe avec trappe à pollen } \\
\hline & $\%$ & $\begin{array}{l}\text { Récolte de miel } \\
\text { par ruche en } \mathrm{kg}\end{array}$ & $\%$ & $\begin{array}{l}\text { Récolte de miel } \\
\text { par ruche en } \mathrm{kg}\end{array}$ \\
\hline 1960 & 100 & 17,2 & 93,2 & 16,0 \\
\hline 1961 & 100 & 7,9 & 131,5 & 9,6 \\
\hline 1962 & 100 & 13,8 & 113,4 & 15,7 \\
\hline
\end{tabular}

Il est évident d'après ces chiffres que la récolte de miel n'est pas affectée par la pose des trappes dans les conditions de l'expérience et que cette récolte peut même être supérieure à celle des témoins. L'auteur conclut donc à l'intérêt pratique de récolter du pollen puisque l'opération est sans incidence défavorable sur la récolte de miel.

Louveaux (1958) a relevé à Bures-sur-Yvette pendant plusieurs années les variations de poids de ruches munies de trappes à pollen pendant toute la saison. En 1953 par exemple cinq ruches ont récolté toutes du miel bien que la région soit assez peu mellifère et l'une d'elle a même atteint un surplus de $16 \mathrm{~kg}$ de miel. Au cours de ces essais, l'auteur a montré qu'aucune relation n'existait entre la récolte du pollen et celle du miel. Les ruches qui amassaient le plus de pollen ne récoltaient pas forcément plus de nectar que les autres.

Ces derniers résultats ne cadrent guère avec les nôtres puisque nous évaluons la perte de rendement en miel à environ $25 \mathrm{p}$. Ioo lorsque des pièges à pollen équipent les ruches, mais les conditions sont peut être différentes. Signalons encore que ROSENTHAL ( I 967 ) en Roumanie a trouvé récemment des résultats différents de ceux de BARAC : la récolte du pollen au moyen des trappes gênerait la récolte du miel dans une certaine mesure.

\section{CONCLUSION}

Il semble difficile de conclure en l'état actuel de nos connaissances, malgré les essais que nous avons effectués. Toutes les expériences réalisées restent encore des cas particuliers et nous ne pouvons pas généraliser les résultats obtenus. Cependant, 
dans les trois essais que nous avons conduits la récolte du pollen a toujours provoqué une diminution du rendement en miel. Le rendement total en miel des colonies est toutefois moins affecté par la pose des trappes que la récolte elle-même. Nous avons vu que cette baisse de rendement en miel atteint près du quart du rendement des témoins. Cependant, dans certaines années favorables (par exemple en 1962) il est quand même possible de recueillir plus de $5 \mathrm{~kg}$ de pollen par ruche en 40 jours, ce qui compense largement la perte de $3,74 \mathrm{~kg}$ de miel. Nous avons constaté que les surfaces de couvain étaient très peu affectées par la pose des pièges à pollen et que les populations des ruches étaient normales en fin d'opération. Nous n'avons pas déterminé quel est le facteur qui fait baisser le rendement en miel des colonies productrices de pollen. Est-ce la trappe elle-même qui cause une gêne aux abeilles ou le manque de pollen? Il est très difficile de le savoir car au moment de la récolte principale du miel il y a longtemps que les trappes sont enlevées ; d'autre part, les colonies ayant eu ces trappes possèdent des réserves de pollen très suffisantes dans leurs rayons. D'après Rybakov, la pose des pièges à pollen augmenterait le pourcentage de butineuses destinées à la récolte du pollen ce qui diminuerait par là même le nombre d'abeilles récoltant le nectar. Mais en ce qui nous concerne, le pollen étant récolté environ 40 à 50 jours avant le début de la grande miellée nous ne pensons pas qu'un déséquilibre dans les activités de la population puisse se maintenir aussi longtemps.

En définitive, dans une région donnée, les apiculteurs désireux de récolter du pollen en grande quantité doivent étudier la rentabilité de l'opération avant de s'y engager. Il est primordial de ne chercher à produire du pollen que dans les régions très pollinifères sans oublier que cette production demande un équipement spécial et beaucoup plus de main d'œuvre et de déplacements que la seule récolte du miel. L'étude de la rentabilité du rucher en général n'est pas encore assez avancée pour qu'on puisse répondre à toutes les questions d'ordre économique qui se posent. Les prix de vente respectifs du pollen et du miel sont également des éléments importants à considérer. Ces prix sont variables mais dans de nombreux cas, la production du pollen peut compléter celle, plus classique, du miel sans nuire gravement au rucher et à son rendement. Il peut même être très intéressant de placer des trappes à pollen pendant toute la saison sur un certain nombre de ruches dans une région où les plantes pollinifères abondent. Nous avons vu que dans ce cas la récolte atteint une dizaine de kilogrammes par ruche et cette quantité amassée par les butineuses compense très largement 1'absence presque totale de récolte de miel.

Reçu pour publication en juillet 1967 .

\section{SUMMARY}

THE EFFECT OF USING POLLEN TRAPS ON THE HONEY YIELD OF BEES COLONIES

This paper is a critical study of collecting pollen by placing traps on hives. The author was interested in the effect of using this method in practical apiculture on the honey yield. Atout a hundred bee colonies were used in southeast France for three years. The main results always showed the same pattern, as shown in Tables $3^{-6}$.

I. Placing pollen traps on the hives for 40 days decreased the honey yield by an average of 24.I p. 100. itself.

2. The total honey yield of the colonies was less affected by traps being set than the harvest 
3. In a good year the weight of pollen harvested compensated for the lowering of the honey yield. In 1962 , for example, the loss of yield in comparison to the controls was $12.8 \mathrm{p}$. roo for a harvest of $5 \mathrm{~kg}$ of pollen per bee colony.

4. In a bad year only a small amount of pollen was harvested and the honey yield was lower than in the controls.

5. Setting pollen traps did not cause any obvious regression of the surfaces of the brood $(4,4$ p. 100 less than in the controls).

Table 7 summarizes the development of the brood during the experiments.

6 . When pollen traps were placed on the hives from March to October, the pollen harvest (about $10 \mathrm{~kg}$ on average) largely compensated for the almost total absence of honey harvest.

Some advice to apiarists is derived from the discussion of the results.

\section{RÉFÉRENCES BIBLIOGRAPHIQUES}

BARAC I., 1965. ('ercetari privind recoltarea polenului en ajutorul albinelor. I.ucrari Stiinfice, 5, 5-14.

BötTcIIER F. K., I9t1. I/3 Zentner Pollen geerntet. Jein neues Pollenernte-gerät. Leipsiger Bienen-Ztg., 56, (2).

Chacyin R., i 955. Rélexions sur la récolte du pollen à l'aide des trappes en 1954. L'apiculteur, i 3-2 I.

FresNaye J., r 962 . In appareil pour le calcul rapide des surfaces de couvain dans les ruches. Ant. Abeille, 5, I 45 -I 53 .

HiRscitfelder HI., I95I. Quantitative Untersuchungen zum Polleneintragen der Bienenvölker. $Z$. Bienen;orsch., 1, 5+, 67-77.

IaviE P., et Fresnaye J., 196.3 . Étude expérimentale de la trappe à pollen en position supérieure. Ann. Abeille, 6, (4), 277-301.

Lovvenux J., i954. Étude sur la récolte du pollen par les abeilles. L'Apiculteur, 43-50.

Locvenux J., 1958. Recherches sur la récolle du pollen par les abeilles (Apis mellifica L.). Thèse Paris.

Rosentual C., I 967 . non publié, communication verbale.

Rybakov M. N., 196I. Pollen traps and the activity of bees. Pchelorodstivo, $38(2), 15^{-1} 6$.

ToDl, F. E., Bislop R. K., 1940. Trapping honeybee-gathered pollen and factors affecting yield. J. econ. Entomol., 33, 866-870. 